\title{
Small in size only
}

Planets and their systems have long held the spotlight, but researchers, space agencies and even the private sector and the public have turned their attention to small bodies.

A stronomy in 2019 started with a bang: the flyby of the farthest body ever visited by a human artefact. The close approach of the New Horizons probe to the recently named Arrokoth, a contact binary Kuiper belt object, allowed scientists to look for the first time at a pristine body, probably undisturbed for the past 4 billion years or so.

We are living in a golden age for small bodies, as various space exploration events of 2019 clearly show. A major theme of the year was sample return. As discussed in a previous Editorial, sample return is more than a curiosity or a technical achievement. Simple remote sensing or in situ analysis are not able to provide the same level of scientific information. NASA's OSIRIS-REx team published their first results from the monitoring of asteroid Bennu and JAXA's Hayabusa 2 performed a series of amazing manoeuvres throughout the year, which included retrieving two samples from asteroid Ryugu's surface, creating an artificial crater on it, and deploying a second rover, MINERVA-II-2 (which failed before deployment but was released anyway in orbit to perform some gravitational measurements).

Among the other exciting small-bodies events of the year, the discovery of the second interstellar object, in September, deserves mention. 2I/Borisov, contrary to $1 \mathrm{I} /$ 'Oumuamua, has a clear cometary character, highlighting the fact that the diversity of small bodies in our Solar System is mirrored in interstellar interlopers. And we were able to detect 2I/Borisov on its way in, before it reached perihelion, so we will be able to acquire data for much longer than we could for 'Oumuamua.

2019 was also a good year for the future of small-bodies exploration. Both Lucy and Psyche, the latest NASA Discovery missions to be selected, passed critical technical reviews and are on course for their expected launch dates in 2021 and 2022, respectively. Both missions target small bodies (a tour of some Jupiter Trojans and the possibly metallic Psyche asteroid, respectively), and in general NASA likes to send their smallbudget missions towards such targets, as discussed by Mike A'Hearn in a Comment.

There is good news from the ESA side too, as it approved the innovative Comet Interceptor mission (actually an ESA/JAXA collaboration), a spacecraft that will lurk at a Lagrange point until a special target - a pristine comet, or maybe an interstellar object - will pass by. The probe will then be ready to approach it and perform close observations. Other agencies are also active: JAXA has laid down a full small-bodies programme (see the Comment by Masaki Fujimoto and Elizabeth Tasker) and China announced plans to launch in 2022 an ambitious mission involving flybys of an asteroid and a comet, with sample return from the asteroid.

One of the main reasons that small bodies have become so popular is their extreme versatility in terms of science exemplified by the content in this issue of Nature Astronomy. In a pair of Letters, Johnson et al. and Zhu et al. study specific phenomena that affected the formation and evolution of asteroids. In particular, Johnson et al. highlight the possible existence of a new kind of volcanism, called ferrovolcanism, that could have created eruptions of metal-rich material from the core to the surface of metallic asteroids like Psyche. Zhu et al's laboratory experiments explain instead how water ice can be continually generated by space weathering on airless bodies. An important component of small-bodies studies is looking at families - groups of dynamically related objects created by some catastrophic impact - as they hold crucial clues on the evolution and history of the asteroid belt. In an Article, Carruba et al. study the very young families, the only ones for which sub-families (groups of bodies generated by subsequent impacts) can be discerned. In their Article, Pike et al. also studied a family, but a specific one: the Haumea family, the only one identified so far in the Kuiper belt. The last paper, a Letter by Guzik et al., analyses the first detailed observations of 2I/Borisov. Finally, the Review Article by Kruijer et al. shows how the isotopic composition of meteorites holds clues on the very first years of the Solar System, with the existence of two nonmixing reservoirs of material (carbonaceous and non-carbonaceous, respectively) that generated the diversity of small bodies we observe today. These papers demonstrate the main role of small bodies in research: they hold unique clues to the understanding of the formation and evolution of the Solar
System, compositionally, structurally and dynamically.

Beyond science, the community is also motivated by planetary defence, for which we need to characterize near-Earth asteroids in order to understand what can be done to avert a catastrophic impact on our planet. Both NASA and ESA are stepping up their efforts, which translates into the approval of new missions. Two spacecraft that had been previously rejected got approved this year. ESA's Hera, a probe that will look at the aftermath of the impact created by the NASA mission DART on asteroid Didymos's moon, got funded in the recent November 2019 ministerial conference, Space19+. The combination of DART and Hera, a project called AIDA, will assess our capability to deflect asteroids in case we need to. From NASA's side, the Near-Earth Object Surveillance Mission (formerly called NEOCam) was finally greenlighted, aimed at monitoring the skies to detect NEOs (usually fast and dark, thus difficult to find with telescopes).

Finally, small bodies are considered as enablers for human exploration. Resource utilization and asteroid mining have started to leave the realms of pure science fiction, being increasingly discussed by scientists, agencies and even the private sector in practical terms. The recent announcement of a Luxembourg Space Resources Innovation Centre demonstrates that the topic is here to stay. Before it can happen, a serious discussion concerning resource exploitation, sustainability and ethical issues will need to be established.

2020 will also be rich in milestones in small-bodies exploration. In the first half of the year OSIRIS-REx will try to acquire a sample from the recently chosen landing site, a place in the northern hemisphere covered in dark regolith called Nightingale. At the very end of the year, Hayabusa 2 will arrive back at Earth with its container of fresh asteroidal material. Interstellar comet Borisov will remain visible for most of the year, probably until September. It's a safe bet that these small bodies will be big on impact in 2020 and beyond.

Published online: 14 January 2020 https://doi.org/10.1038/s41550-019-1006-6 